\title{
Robust quantitative assessments of cytosine modifications and changes in the expressions of related enzymes in gastric cancer
}

\author{
Chunping Du $\cdot$ Nobuya Kurabe $\cdot$ Yoshitaka Matsushima $\cdot$ Masako Suzuki $\cdot$ Tomoaki Kahyo \\ Ippei Ohnishi · Fumihiko Tanioka $\cdot$ Shogo Tajima $\cdot$ Masanori Goto $\cdot$ Hidetaka Yamada • \\ Hong Tao $\cdot$ Kazuya Shinmura $\cdot$ Hiroyuki Konno $\cdot$ Haruhiko Sugimura
}

Received: 17 April 2014/Accepted: 13 July 2014/Published online: 7 August 2014

(C) The International Gastric Cancer Association and The Japanese Gastric Cancer Association 2014

\begin{abstract}
Background The rediscovery of 5-hydroxymethylcytosine, the ten-eleven translocation (TET) family, thymineDNA glycosylase $(T D G)$ and isocitrate dehydrogenase $(I D H)$ have opened new avenues in the study of DNA demethylation pathways in gastric cancer (GC). We performed a comprehensive and robust analysis of these genes and modified cytosines in gastric cancer.

Methods Liquid chromatography mass spectrometry/ mass spectrometry (LC-MS/MS) was used to assess 5-methyldeoxycytidine (5-mC), 5-hydroxymethyldeoxycytidine (5-hmC), 5-formyldeoxycytidine (5-fC) and
\end{abstract}

C. Du and N. Kurabe contributed equally to this work.

Supplementary electronic material The online version of this article (doi:10.1007/s10120-014-0409-4) contains supplementary material available to authorized users.

C. Du · N. Kurabe $\cdot$ T. Kahyo · I. Ohnishi - S. Tajima ·

M. Goto $\cdot$ H. Yamada $\cdot$ H. Tao $\cdot$ K. Shinmura .

H. Sugimura $(\square)$

Department of Tumor Pathology, Hamamatsu University School of Medicine, 1-20-1 Handayama, Higashi Ward, Hamamatsu,

Shizuoka 431-3192, Japan

e-mail: hsugimur@hama-med.ac.jp

Y. Matsushima

Department of Chemistry, Hamamatsu University School of

Medicine, Shizuoka, Japan

Present Address:

Y. Matsushima

Department of Applied Biology and Chemistry, Faculty

of Applied Bioscience, Tokyo University of Agriculture,

1-1-1 Sakuragaoka, Setagaya-ku, Tokyo 156-8502, Japan

M. Suzuki

Research Equipment Center, Hamamatsu University School of

Medicine, Shizuoka, Japan 5-carboxyldeoxycytidine (5-caC) quantitatively in tumorous and non-tumorous regions of $\mathrm{GCs} ;\left[\mathrm{D}_{2}\right]-5-\mathrm{hmC}$ was used as an internal standard. Expression levels of the genes TET1, TET2, TET3, TDG, IDH1 and IDH2 were measured using a real-time reverse transcription polymerase chain reaction (RT-PCR) and were compared to the clinical attributes of each case. Using HEK293T cells the effects of introducing plasmids containing full-length TET1, TET2, and TET3 and 7 variants of the TET2 catalytic domain were evaluated in terms of their effect on cytosine demethylation.

Results LC-MS/MS showed that 5-hmC was significantly decreased in tumorous portions. 5-mC was also moderately decreased in tumors, while 5-fC and 5-caC were barely detectable. The expressions of TET1, TET2, TET3, TDG and $I D H 2$, but not $I D H 1$, were notably decreased in GCs, compared with the adjacent non-tumor portion. TETI

I. Ohnishi · F. Tanioka

Iwata City Hospital, Iwata, Shizuoka, Japan

S. Tajima

Shizuoka Saiseikai Hospital, Shizuoka, Japan

Present Address:

M. Goto

Division of Cancer Development System, National Cancer

Center Research Institute, 5-1-1 Tsukiji, Chuo-ku,

Tokyo 104-0045, Japan

H. Konno

Department of Surgery II, Hamamatsu University School of Medicine, Shizuoka, Japan 
expression and the 5-hmC levels determined using LC-MS/ MS had a significantly positive correlation and TET1 protein had a greater effect on the increase in $5-\mathrm{hmC}$ than TET2 and TET3 in HEK293T cells.

Conclusions The loss of 5-hmC and the down-regulation of TET1-3, TDG and IDH2 were found in GCs. The loss of 5 -hmC in GCs was mainly correlated with the down-regulation of TETI.

Keywords Cytosine modification - Liquid chromatography-mass spectrometry $\cdot\left[\mathrm{D}_{2}\right]-5-\mathrm{hmC}$. Ten-eleven translocation family genes $\cdot$ Adductomics

\section{Introduction}

The ten-eleven translocation (TET) family proteins (TET1, TET2 and TET3) are responsible for the oxidation of 5-methylcytosine (5-mC) in DNA to 5-hydroxymethylcytosine $(5-\mathrm{hmC})$ and the further oxidation of $5-\mathrm{hmC}$ to 5-formylcytosine (5-fC) and 5-carboxylcytosine (5-caC), which are then cleaved from the DNA using thymineDNA glycosylase (TDG) to be replaced with unmethylated cytosine [1-4]. Additionally, the oxidation reaction of TET proteins is dependent on $\mathrm{Fe}^{2+}$ and 2-oxoglutarate (2-OG), and 2-OG is dependent on the activity of isocitrate dehydrogenase IDH1 and IDH2 [5, 6] (Figure S1). The loss of 5-hydroxymethylcytosine and the down-regulation of the TET family and $I D H 2$ have been found in human cancers [7-11], but comprehensive evaluations of the modified cytosines and the enzymes that are involved have remained largely unperformed and most previous studies have used dot blot analyses as a means of measuring 5-hydroxymethyldeoxycytidine (5-hmC), which is at best a semi-quantitative method. Because dot blot analysis is based on the antigen-antibody reaction direct assessments of the absolute amount of 5-mC, 5-hmC, 5-fC, and 5-caC are not possible. Moreover, since the signal intensities of dot blot images become easily saturate, the standard curve used in the analyses is not linear [12]. In contrast to dot blot analysis, liquid chromatography coupled with tandem mass spectrometry (LC-MS/ MS) can calculate the absolute quantity of the modifications and its standard curve is completely linear. Therefore, LC-MS/MS has greater sensitivity than dot blot analysis. Hence, we established an assay system using LC-MS/MS using a stable isotope standard to measure 4 modified deoxycytidines at the same time and these values were compared to the expression profiles of TET1-3, $T D G$ and IDHI-2.

In this study we applied LC-MS/MS to assess 5-caC, 5-fC, 5-hmC and 5-mC in the genomic DNA of tumor portions and the corresponding non-tumor portions of gastric cancer (GC) samples. TETI-3, TDG and IDHI-2 were quantitated using a real-time reverse transcriptionpolymerase chain reaction (RT-PCR). Next the coding exons of TET1-3 were analyzed by sequencing to search for possible somatic (tumor-specific) mutations and polymorphisms with neural or unknown functionality. Then we analyzed the effect of 5-mC oxidation in vitro by the introduction of TET1-3 and 7 TET2 variants into immortalized human embryonic kidney cells (HEK293T).

\section{Materials and methods}

Tissue samples and patients

GC tissues and the corresponding adjacent non-tumorous tissues from a total of 58 sporadic GC cases were obtained from the Hamamatsu University Hospital in Japan. The mean patient age was 66.9 years (SD 11.9 years) and the patients included 35 men and 23 women with 26 intestinal and 32 diffuse histological types. All cases were clinically operable and the stages ranged from I to III (no stage IV cases were included). Resected tissues, which were verified as tumorous regions $(>70 \%)$ or non-tumorous regions (no contamination with tumor cells) under a microscope by attending pathologists, were snap-frozen in liquid nitrogen and stored at $-80{ }^{\circ} \mathrm{C}$ until used for the isolation of DNA and RNA. Histological classifications were based on the Japanese Classification of Gastric Carcinoma [13] and Lauren's classification [14] was applied when the cases were categorized into two types.

For comparison 5 pairs of gastric cancers and matched normal tissues from "Gan mice" (K19-Wnt1/C2mE), a kind gift of Prof. Masanobu Oshima at the Kanazawa University, were also used to measure these cytosine modifications in a non-human model of gastric cancer [15]. The project was approved by the Institutional Review Board of the Hamamatsu University School of Medicine (23-91).

Synthesis of stable isotope-labeled $\mathrm{hmC}$ and standard chemicals

Stable isotope-labeled 5-hydroxymethyl-2'-deoxycytidine $\left(\left[\mathrm{D}_{2}\right]-5-\mathrm{hmC}\right)$ was synthesized from commercially available 5-iodo-2'-deoxycytidine (TCI, Tokyo Chemical Industry Co., Ltd., Tokyo, Japan) according to the literature [16]. 5-caC was also synthesized from commercially available 5-iodo-2'-deoxycytidine according to a previously reported protocol $[17,18]$. 5-fC and 5-hmC were also synthesized from commercially available 5-mC (TCI) according to a previously reported protocol [19]. 
DNA purification and hydrolysis for LC-MS/MS

Genomic DNA was isolated and purified from samples of human gastric cancer. Approximately $100 \mathrm{mg}$ of samples were homogenized with $600 \mu$ of lysis solution $(10 \mathrm{mM}$ Tris-HCl [pH 8.0], 5 mM EDTA, $0.5 \%$ SDS, and $0.01 \%$ deferoxamine mesylate) using a Physcotron Handy Homogenizer NS-310EII (Microtech nichion, Chiba, Japan) at 15,000 rpm for $2 \mathrm{~min}$. The homogenates were treated with $15 \mu \mathrm{l}$ of protease K (QIAGEN, CA, USA) at $55^{\circ} \mathrm{C}$ for $3 \mathrm{~h}$ and $15 \mu \mathrm{l}$ of RNase A (QIAGEN) at $37^{\circ} \mathrm{C}$ for $40 \mathrm{~min}$. Two hundred microliters of protein precipitation solution (1 mM EDTA, $8 \mathrm{M}$ ammonium acetate, $0.01 \%$ deferoxamine mesylate) was added to the mixture followed by centrifugation at $14,000 \mathrm{~g}$ in room temperature for 5 min to remove proteins. Six hundred microliters of isopropanol was added to the supernatant and then centrifuged at $14,000 \mathrm{~g}$ in room temperature for $5 \mathrm{~min}$ to precipitate the genomic DNA. Precipitates were washed with $70 \%$ ethanol and dried using a Speed-Vac concentrator (Savant, NY, USA). The dried genomic DNA was hydrated with distilled water at the concentration of about $1 \mathrm{mg} / \mathrm{ml}$. Five micrograms of genomic DNA were treated with $50 \mathrm{U}$ of DNaseI (New England Biosystems, MA, USA) for $30 \mathrm{~min}$ at $37{ }^{\circ} \mathrm{C}$. After incubation $5 \mu \mathrm{g}$ of phosphodiesterase I, $10 \mathrm{U}$ of calf intestinal alkaline phosphatase (TaKaRa, Kyoto, Japan) and $10 \mathrm{ng}$ of [ $\left.\mathrm{D}_{2}\right]-5-\mathrm{hmC}$ were added to the mixture. After $1 \mathrm{~h}$ of incubation at $37^{\circ} \mathrm{C}$ the proteins were removed using Nanosep 3K Omega (Pall Corp., MI, USA) by centrifugation at $14,000 \mathrm{~g}$ in $4{ }^{\circ} \mathrm{C}$ for $20 \mathrm{~min}$. Digested samples were stored at $-30^{\circ} \mathrm{C}$ until used for LC-MS/MS analysis.

\section{Quantification using LC-MS/MS}

LC-MS/MS analyses were performed using a 4000 QTRAP mass spectrometer with an Acquity ${ }^{\mathrm{TM}}$ Ultra Performance LC system (AB SCIEX Instruments, Foster City, CA, USA). Digested DNA was injected and separated using an Acquity UPLC HSS T3 column $(2.1 \mathrm{~mm} \times 100 \mathrm{~mm}$; Waters, Milford, CT, USA). The column was eluted as follows: deoxycytidine (C) and 5-mC were eluted in $94 \%$ $\mathrm{B}$ from 0 to $3 \mathrm{~min}$ and in a linear gradient of 6-60\% B from 3 to $8 \mathrm{~min}$ at $0.2 \mathrm{~mL} / \mathrm{min} ; 5-\mathrm{hmC}$ was eluted in $6 \% \mathrm{~B}$ from 0 to $3 \mathrm{~min}$ and $20 \% \mathrm{~B}$ from 3 to $8 \mathrm{~min}$ at $0.2 \mathrm{~mL} / \mathrm{min}$ (mobile phase A: $0.02 \%(\mathrm{v} / \mathrm{v})$ acetic acid in water; mobile phase B: methanol); and 5-fC and 5-caC were eluted in 94 $\% \mathrm{~B}$ from 0 to $3 \mathrm{~min}$ and in a linear gradient of $6-20 \% \mathrm{~B}$ from 3 to $10 \mathrm{~min}$ at $0.2 \mathrm{~mL} / \mathrm{min}$. Multi-reaction monitoring was performed in positive ion mode using nitrogen as the nebulizing gas. Experimental conditions were as follows: ion source temperature, $400^{\circ} \mathrm{C}$; cone voltage, $5500 \mathrm{~V}$; collision gas, nitrogen. Collision energies and characteristic reactions were as follows (collision energy $(\mathrm{eV})$, base isonS $\rightarrow$ product ion): $\left[\mathrm{D}_{2}\right]-5-\mathrm{hmC}(13 \mathrm{eV}$, $260.076 \rightarrow 144.000), \mathrm{C}(13 \mathrm{eV}, 228.045 \rightarrow 111.900), 5-\mathrm{mC}$ $(15 \mathrm{eV}, 242.114 \rightarrow 126.100), 5-\mathrm{hmC}(13 \mathrm{eV}, 258.044 \rightarrow$ $142.000), 5-\mathrm{fC}(256.039 \rightarrow 140.0)$ and 5 -caC $(272.000 \rightarrow$ 156.000). C, 5-mC, 5-hmC, 5-fC and 5-caC were quantified by calculating the peak area ratio of the analytes and $\left[\mathrm{D}_{2}\right]-5$-hmC. Calibration curves were drawn by measuring the amount of authentic standards spiked with $\left[\mathrm{D}_{2}\right]-5$ $\mathrm{hmC}$. The numbers of $\mathrm{C}, 5-\mathrm{mC}$ and $5-\mathrm{hmC}$ were calculated by dividing by the moles of total nucleosides.

Analysis of TET1-3, TDG and IDH1-2 transcript levels

Details are provided in the supplementary materials (Table S1).

Sequencing analysis

Details are provided in the supplementary materials.

Plasmid construction

Details are provided in the supplementary materials.

Cell culture and transfection

Details are provided in the supplementary materials.

Western blot analysis

Details are provided in the supplementary materials.

DNA dot blot analysis

Details are provided in the supplementary materials.

Statistical analysis

Details are provided in the supplementary materials.

\section{Results}

Global 5-mC and 5-hmC levels in GCs

We used the LC-MS/MS method for the quantification of 5-mC, 5-hmC, 5-fC and 5-caC. $\left[\mathrm{D}_{2}\right]-5-\mathrm{hmC}$ was used as an internal standard. Calibration curves were drawn by measuring the amount of authentic standards spiked with $\left[\mathrm{D}_{2}\right]-$ 5-hmC (Figure S2). Detection limits for the column were as follows: 5-mC, $0.059 \mathrm{fmol}$; 5-hmC, $0.057 \mathrm{fmol}$; 5-fC, $0.103 \mathrm{fmol}$; and 5-caC, $0.133 \mathrm{fmol}$. 5-fC and 5-caC were 
excluded from subsequent analyses because we could not detect any signal peaks above the detection limits. The resulting calibration curve was used to determine the global levels of C, 5-mC and 5-hmC in 28 pairs of GC tissues. The $5-\mathrm{mC}$ and $5-\mathrm{hmC}$ values were quantified with reference to the quantity of $\mathrm{C}$. As shown in Table S2, 5-mC values ranged from 292.00 modifications per $10^{4} \mathrm{C}$ base (292.00/ $10^{4} \mathrm{C}$ ) to $430.62 / 10^{4} \mathrm{C}$, while the 5 -hmC values ranged from $1.63 / 10^{4} \mathrm{C}$ to $19.54 / 10^{4} \mathrm{C}$. The median $5-\mathrm{mC}$ value was $377.92 / 10^{4} \mathrm{C}$ in the normal tissues and $366.66 / 10^{4} \mathrm{C}$ in the cancer tissues, while the median 5 -hmC value was 9.63 / $10^{4} \mathrm{C}$ in the normal tissues and $3.32 / 10^{4} \mathrm{C}$ in the cancer tissues. In total 5-mC was moderately decreased in the tumor portions compared with the normal portions (Table S2, $P=0.021) .5$-hmC was strikingly decreased in the tumor portions of the GC cases (Table S2, $P<0.001$ ). The reduction of 5-hmC in the tumor portion was correlated with the $\mathrm{N}$ classification (Table $1, P=0.018$ ). No correlation was seen between the reduction of 5-mC in the tumor portion and any of the clinicopathological factors that were examined (Table 1).

For comparison, in terms of the reduction of $5-\mathrm{hmC}$ in the tumor portion, we also performed the same analysis using 5 pairs of gastric cancers and adjacent gastric mucosa from the so-called Gan mouse (K-19-Wnt1/C2mE mouse), which has been established as an inflammation-mediated rodent stomach cancer model [15]. The number of samples that were examined was too small for differences in the $5-\mathrm{mC}$ and 5-hmC values between the tumor portion and the non-tumor portion to reach a statically significant level. However, the 5-hmC level seemed to be lower in the tumorous portion (Figure S3).

Quantification of TET1-3, TDG and IDH1-2 transcripts in GCs

Expressions of TET1-3, TDG and $I D H 2$ were significantly decreased in the tumor portions of the GC cases compared
Table 1 Comparisons of 5-mC and 5-hmC levels in gastric cancer tissues with adjacent normal tissues of GC patients in different clinicopathological factors
A two-tailed independent t-test was used when the number of compared groups is 2. ANOVA testing was used when the number of compared groups is more than 3. $P<0.05$ was considered statistically significant. N: normal tissue; T: tumor tissue. Indicated are the mean of $\mathrm{T}$ divided by $\mathrm{N}$, and $\mathrm{SE}$

a 4 cases in which the tumor covered all of the lower, middle and upper parts of the stomach

\begin{tabular}{|c|c|c|c|c|c|c|c|}
\hline \multirow[t]{2}{*}{ Characteristics } & \multirow[t]{2}{*}{ Cases } & \multicolumn{3}{|l|}{$5-\mathrm{mC}$} & \multicolumn{3}{|l|}{ 5-hmC } \\
\hline & & Mean $(\mathrm{T} / \mathrm{N})$ & SE & $P$ value & Mean $(\mathrm{T} / \mathrm{N})$ & SE & $P$ value \\
\hline \multicolumn{8}{|l|}{ Age } \\
\hline$>60$ & 24 & 0.950 & 0.021 & \multirow[t]{2}{*}{0.286} & 0.344 & 0.031 & \multirow[t]{2}{*}{0.248} \\
\hline$\leq 60$ & 4 & 1.008 & 0.013 & & 0.696 & 0.246 & \\
\hline \multicolumn{8}{|l|}{ Gender } \\
\hline Male & 17 & 0.980 & 0.252 & \multirow[t]{2}{*}{0.162} & 0.356 & 0.038 & \multirow[t]{2}{*}{0.322} \\
\hline Female & 11 & 0.926 & 0.250 & & 0.454 & 0.105 & \\
\hline \multicolumn{8}{|l|}{ Location } \\
\hline Lower & 8 & 0.917 & 0.026 & \multirow[t]{4}{*}{0.473} & 0.373 & 0.048 & \multirow[t]{4}{*}{0.127} \\
\hline Middle & 4 & 0.981 & 0.015 & & 0.444 & 0.090 & \\
\hline Upper & 12 & 0.958 & 0.032 & & 0.284 & 0.037 & \\
\hline Others $^{\mathrm{a}}$ & 4 & - & - & & - & - & \\
\hline \multicolumn{8}{|l|}{$\mathrm{T}$ classification } \\
\hline $\mathrm{T} 1$ & 5 & 0.937 & 0.032 & \multirow[t]{4}{*}{0.947} & 0.190 & 0.027 & \multirow[t]{4}{*}{0.054} \\
\hline $\mathrm{T} 2$ & 4 & 0.970 & 0.015 & & 0.409 & 0.097 & \\
\hline $\mathrm{T} 3$ & 10 & 0.968 & 0.044 & & 0.350 & 0.049 & \\
\hline $\mathrm{T} 4$ & 9 & 0.955 & 0.028 & & 0.551 & 0.111 & \\
\hline \multicolumn{8}{|l|}{$\mathrm{N}$ classification } \\
\hline No & 10 & 0.994 & 0.035 & \multirow[t]{4}{*}{0.523} & 0.298 & 0.048 & \multirow[t]{4}{*}{0.018} \\
\hline N1 & 2 & 0.936 & 0.006 & & 0.296 & 0.061 & \\
\hline $\mathrm{N} 2$ & 6 & 0.922 & 0.054 & & 0.272 & 0.032 & \\
\hline N3 & 10 & 0.950 & 0.021 & & 0.585 & 0.098 & \\
\hline \multicolumn{8}{|l|}{ Stage } \\
\hline I & 5 & 0.964 & 0.022 & \multirow[t]{3}{*}{0.679} & 0.185 & 0.024 & \multirow[t]{3}{*}{0.070} \\
\hline II & 7 & 0.985 & 0.054 & & 0.366 & 0.050 & \\
\hline III & 16 & 0.945 & 0.022 & & 0.473 & 0.072 & \\
\hline \multicolumn{8}{|l|}{ Histological type } \\
\hline Intestinal & 16 & 0.952 & 0.027 & \multirow[t]{2}{*}{0.688} & 0.378 & 0.039 & \multirow[t]{2}{*}{0.699} \\
\hline Diffuse & 12 & 0.967 & 0.026 & & 0.416 & 0.100 & \\
\hline
\end{tabular}


(a)

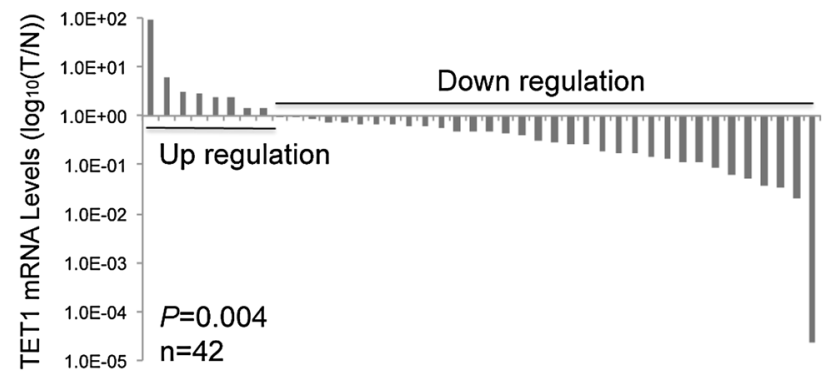

(c)

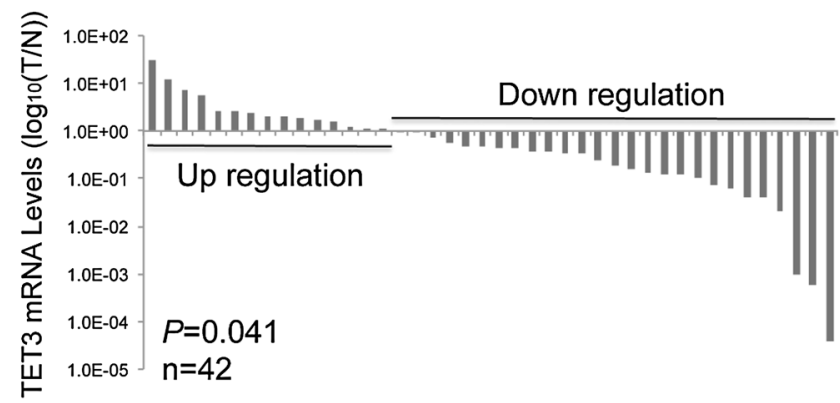

(e)

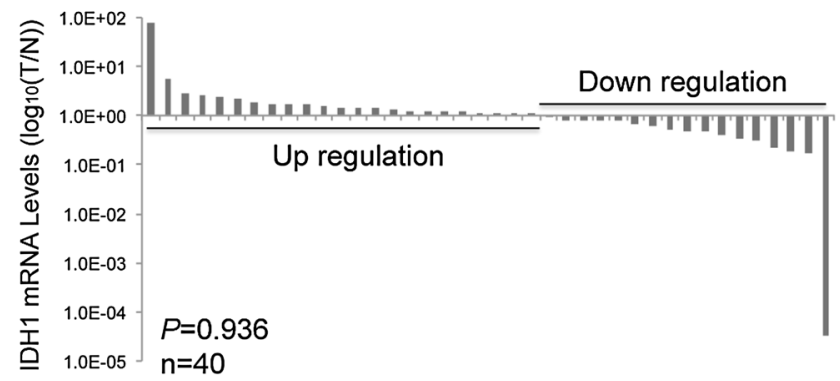

Fig. 1 Gene expression levels in gastric cancer tissue. Measurement of TET1 (a), TET2 (b), TET3 (c), TDG (d), IDH1 (e) and IDH2 (f) mRNA expression levels in GCs and adjacent normal samples

to the corresponding non-tumor tissue (Fig. 1, TET1, $P=$ 0.004; TET2, $P=0.000295 ;$ TET3, $P=0.041 ; T D G, P=$ $0.006 ; I D H 2, P=0.000475$ ) while the expression of $I D H 1$ was not significantly decreased $(P=0.936)$. Down-regulation of TETI in the GCs was correlated with the tumor location (Table S3, $P=0.036$ ). Furthermore, down-regulation of $I D H 2$ was positively correlated with the tumor classification $\mathrm{N}(P=0.002)$ and the tumor stage $(P=0.027)$ (Table 2). However, no significant association between TET2, TET3, TDG or IDH1 down-regulation and any of the clinicopathological factors was seen in our samples (data not shown).

5-hmC levels are correlated with TET1 gene expression

A linear regression analysis showed that TET1 expression was significantly and positively correlated with 5 -hmC

\section{(b)}

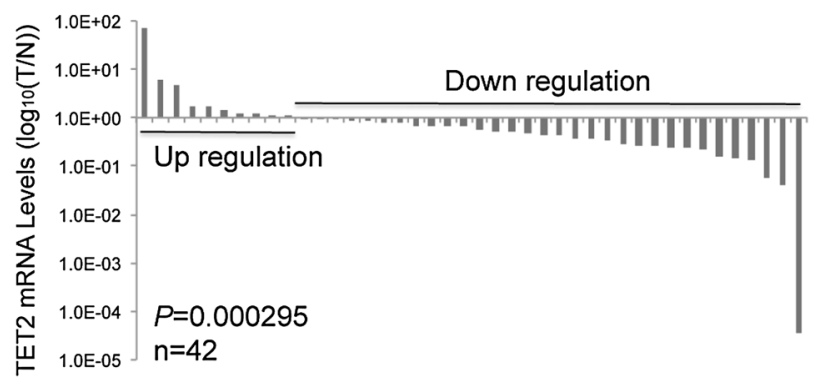

(d)

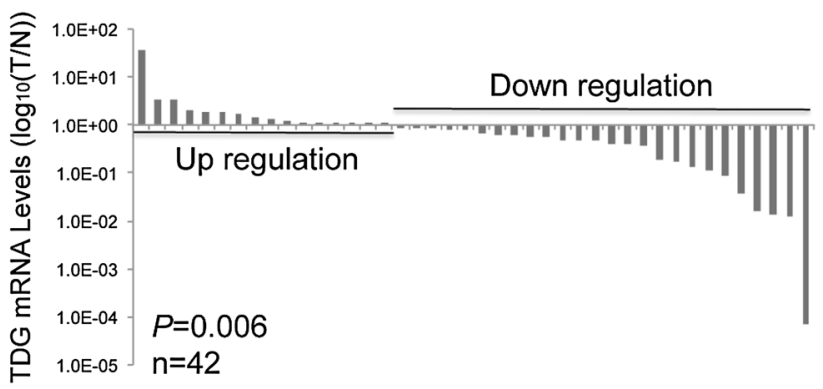

\section{(f)}

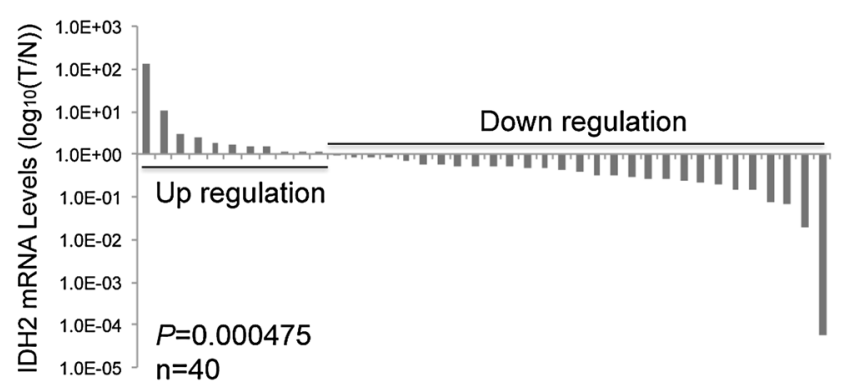

using real-time RT-PCR. $P$ values were analyzed using the MannWhitney $U$ test. $P<0.05$ was considered statistically significant

levels $(P=0.037, \mathrm{R}=0.428$; Pearson's correlation coefficient). However, no correlation was seen between 5-hmC levels and the expressions of TET2, TET3, TDG, IDH1 or $I D H 2$ in the gastric mucosa (Fig. 2).

Comparison of 5-mC oxidation activities of TET1, TET2 and TET3 proteins in vitro

Expressions of TET1-3 were significantly decreased in GCs but only TET1 expression was notably correlated with 5-hmC levels. To further examine the relationships between 5-hmC and TET proteins in human cells we compared the 5-mC oxidation activities of the TET1, TET2 and TET3 proteins. Thus, full-length TET1, TET2 and TET3 plasmids were transfected in HEK293T cells. Then LC-MS/MS and real-time RT-PCR were used to quantitate the 5-hmC and gene expression levels in these cell lines. 
Table 2 Correlation between IDH2 gene expression and clinicopathological attributes

\begin{tabular}{|c|c|c|c|c|}
\hline Variables & Cases & Down-regulation & Up-regulation & $P$-value \\
\hline \multicolumn{5}{|l|}{ Age } \\
\hline$<60$ & 6 & 5 & 1 & \multirow[t]{2}{*}{1.000} \\
\hline$>60$ & 34 & 24 & 10 & \\
\hline \multicolumn{5}{|l|}{ Gender } \\
\hline Male & 27 & 21 & 6 & \multirow[t]{2}{*}{0.451} \\
\hline Female & 13 & 8 & 5 & \\
\hline \multicolumn{5}{|c|}{ Tumor location } \\
\hline Lower & 8 & 4 & 4 & \multirow[t]{4}{*}{0.378} \\
\hline Middle & 14 & 10 & 4 & \\
\hline Upper & 10 & 8 & 2 & \\
\hline Others* & 8 & - & - & \\
\hline \multicolumn{5}{|c|}{$\mathrm{T}$ classification } \\
\hline $\mathrm{T} 1$ & 9 & 5 & 4 & \multirow[t]{4}{*}{0.568} \\
\hline $\mathrm{T} 2$ & 4 & 3 & 1 & \\
\hline $\mathrm{T} 3$ & 15 & 11 & 4 & \\
\hline $\mathrm{T} 4$ & 12 & 10 & 2 & \\
\hline \multicolumn{5}{|c|}{$\mathrm{N}$ classification } \\
\hline No & 13 & 7 & 6 & \multirow[t]{4}{*}{0.002} \\
\hline N1 & 5 & 1 & 4 & \\
\hline $\mathrm{N} 2$ & 10 & 10 & 0 & \\
\hline N3 & 12 & 11 & 1 & \\
\hline \multicolumn{5}{|l|}{ Stage } \\
\hline I & 10 & 5 & 5 & \multirow[t]{3}{*}{0.027} \\
\hline II & 9 & 5 & 4 & \\
\hline III & 21 & 19 & 2 & \\
\hline \multicolumn{5}{|c|}{ Histological type } \\
\hline Intestinal & 23 & 18 & 5 & \multirow[t]{3}{*}{0.285} \\
\hline Diffuse & 15 & 9 & 6 & \\
\hline Mixed & 2 & - & - & \\
\hline
\end{tabular}

$P$ values were evaluated using the Pearson chi-square or the Fisher exact test. $P<0.05$ was considered statistically significant. $* 8$ cases in which the tumor covered all the lower, middle, and upper parts. Down-regulation: Tumor/Normal $<1.0$; Up-regulation: Tumor/Normal $>1.0$

As shown in Fig. 3a, the forced expressions of TETI and TET2 significantly increased the 5-hmC level $(P<0.01)$ while TET3 moderately increased the 5-hmC level. Notably, TET1 had the highest efficiency in global DNA 5-mC oxidation among the TET family proteins.

Sequencing analysis of TETI-3 in the GCs

We were interested in whether 5 -hmC reduction in tumors was associated with the somatic mutation of TETI-3 in GCs. Therefore, sequencing was performed to analyze 18 pairs of GCs. All the GC samples were confirmed to have significantly decreased 5-hmC levels in the tumor portions as compared to the adjacent non-tumor portions when examined using LC-MS/MS.

According to the Japanese Single Nucleotide Polymorphism (JSNP) database and the National Center for Biotechnology (NCBI) (updated on January 31, 2014), 6 SNPs

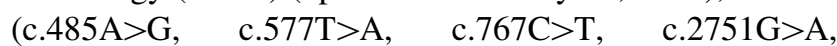
c.3053A $>\mathrm{G}$, and c.3369A $>\mathrm{G})$ in TET1, 6 SNPs $($ c.86C $>\mathrm{G}$, c. $652 \mathrm{G}>\mathrm{A}$, c. $3117 \mathrm{G}>\mathrm{A}, \quad$ c. $4140 \mathrm{~T}>\mathrm{C}, \quad$ c. $5162 \mathrm{~T}>\mathrm{G}$, and c.5284A $>$ G) in TET2, and 2 SNPs (c.1520C $>\mathrm{G}$ and c.2131T $>$ C) in TET3 are currently known. Using a sequencing analysis we found 2 known missense variations (c.5162T $>\mathrm{G}(\mathrm{p} . \mathrm{L} 1721 \mathrm{~W})$ and c.5284A $>\mathrm{G}[\mathrm{p} . \mathrm{I} 1762 \mathrm{~V}])$ in the TET2 catalytic domain and 1 novel variation (c.3000C $>$ T) in TET3. No somatic tumor-specific mutations were found in any of our samples. Two of these SNPs (c.3117G $>$ A and c.4140T $>$ C) in TET2 were previously detected among Japanese children with essential thrombocythemia [20] but their pathogenic significance was unclear. Since our sequencing study was performed using a relatively small number of available samples we decided to include the known functional variants Y1148* [21] and F1706fs*13 [22] in our transfection experiments.

Comparing 5-mC oxidation activity of 7 human TET2 variant proteins

We constructed and tested the 5-hmC-generating activities of TET2 catalytic domain (1123-2002 aa) variants in HEK293T cells identified in this study or in previous studies [21-23]. As shown in Fig. 3b, c, using the DNA dot blot and LC-MS/MS (Figure S4), TET2 proteins obviously increased the number of 5-hmC adducts in human cells. The 5-mC oxidation activities of Y1148* [21] and F1706fs*13 [22] proteins were severely impaired but the functions of A1476V [21], V1836M [21], L1240V [23], L1721W (newly found in this study) and I1762V (newly found in this study) proteins seemed to be retained. Moreover, LC-MS/MS data seems to be consistent with the results obtained from the dot blot analysis.

\section{Discussion}

Herein we applied LC-MS/MS for the quantification of 5-mC, 5-hmC, 5-fC, and 5-caC levels in both the tumorous and non-tumorous portions of human gastric mucosa resected. 5-mC was moderately decreased in GCs. 5-caC and 5-fC are also important metabolites of these dynamics of cytosine modification, as shown in Figure S1. Considering reports on the existence of these molecules are limited in normal brain and brain tumor tissues [24, 25] our attempt was to first try to detect these modified cytosines in human non-neural tissue. We assumed that our detection limits 

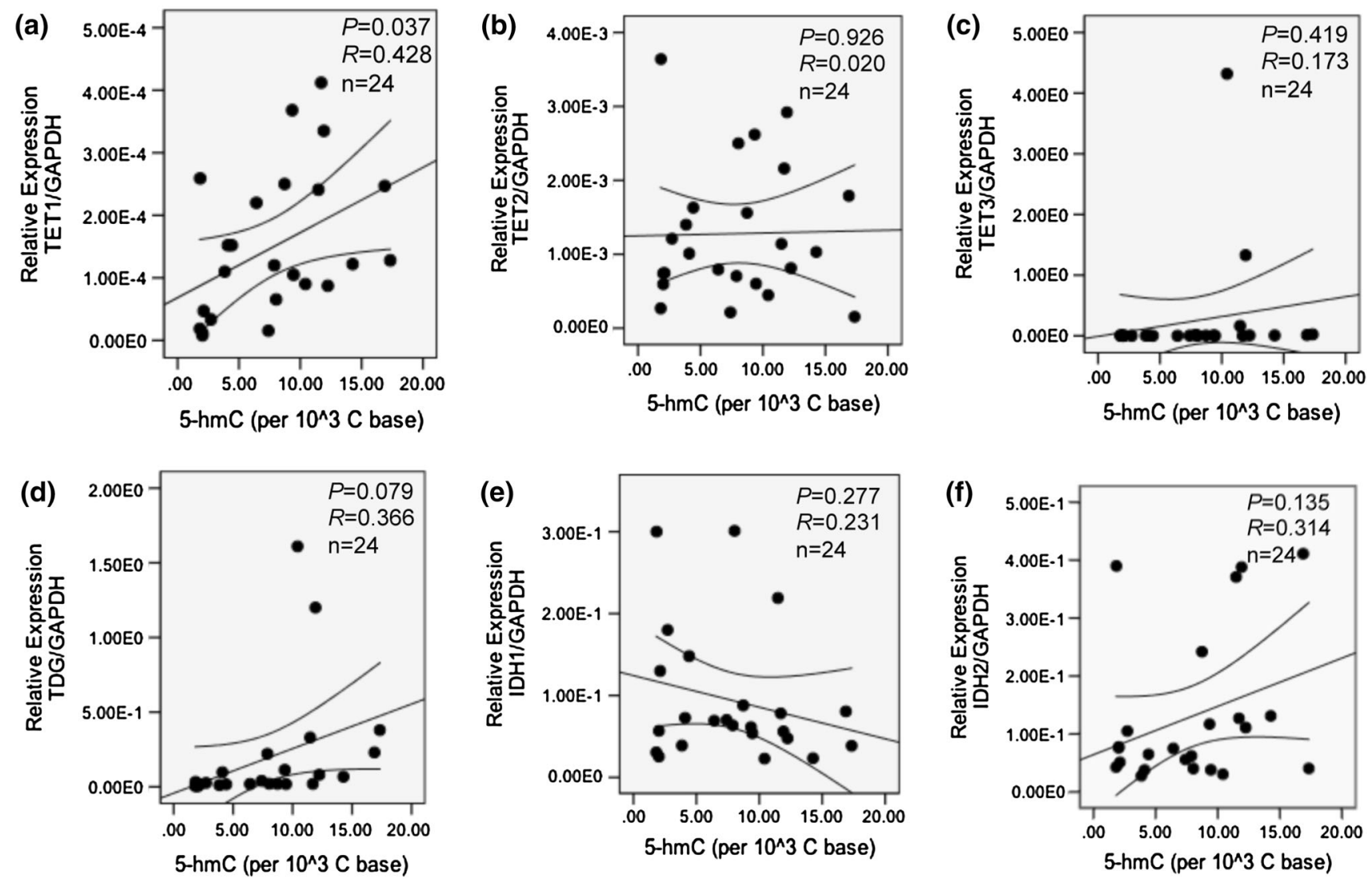

Fig. 2 Linear regression analyzing the relationships between 5-hmC levels and TET1 (a), TET2 (b), TET3 (c), TDG (d), IDH1 (e) and $I D H 2$ (f) mRNA expressions in GCs and adjacent normal samples.
$P$ values were analyzed using a two-tailed Pearson correlation. $P<$ 0.05 was considered statistically significant (a)

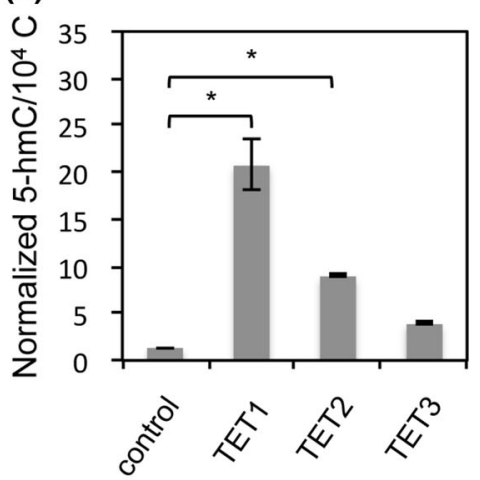

(b)

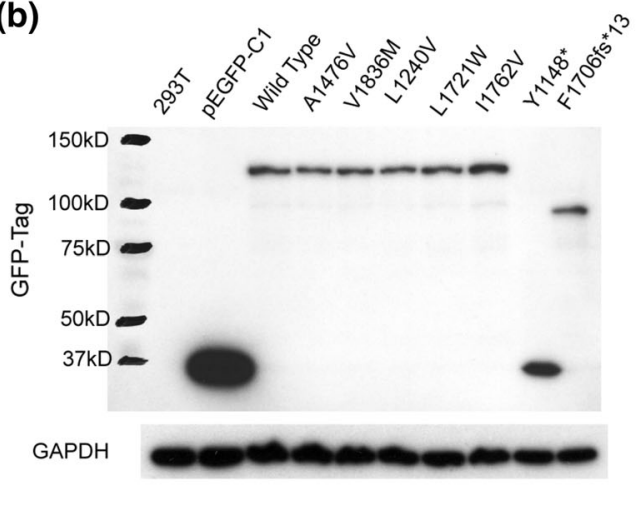

(c)

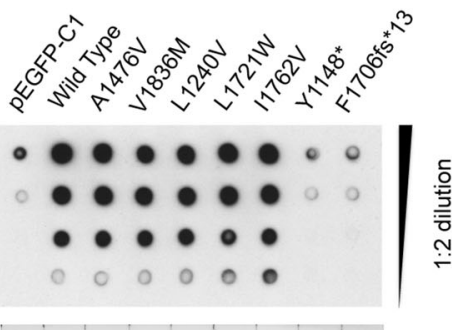

MB
Fig. 3 Overexpression of TET proteins in HEK293T cells. (a) Comparison of 5-mC oxidation activity of TET proteins using LC-MS/MS. 5 -hmC values were divided by the corresponding gene mRNA levels and the standard error. One-way ANOVA with Tukey's post-hoc test, $* P<0.01$; (b) Western blot for detecting the expression of 7 variants

were $0.103 \mathrm{fmol}(5-\mathrm{fC})$ and $0.133 \mathrm{fmol}(5-\mathrm{caC})$ based on calibration curves using internal controls; actually, no signal peaks were observed in human gastric tissues. Thus, we interpreted that there were no 5-fC and 5-caC in human of GFP-tagged TET2 catalytic domain proteins (1123-2002 aa). GAPDH was used as a control; (c) Comparison of 5-mC oxidation activities of 7 variants of TET2 catalytic domain proteins using a DNA dot blot. Methylene blue staining was used as a total genomic DNA loading control. MB: methylene blue

gastric tissues. On the other hand, 5-hmC was reduced by about 3-fold in the tumors of GC cases. In line with recent studies $[9,26]$, our data also show that $5-\mathrm{hmC}$ values varied widely among individuals. 

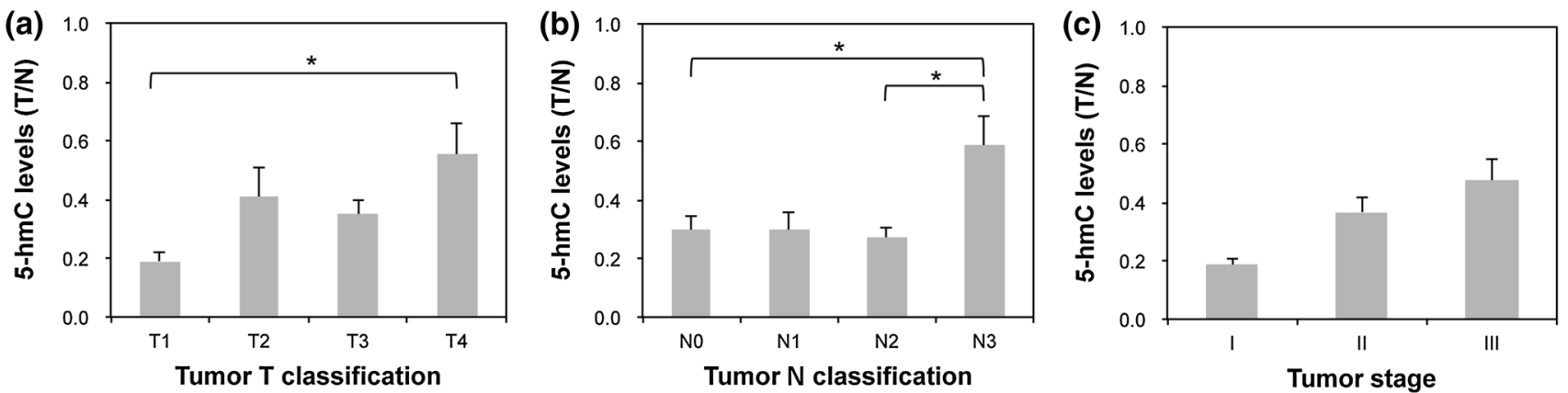

Fig. 4 Comparisons of LC-MS/MS values of 5-hmC in GC tissues and adjacent normal tissues of GC patients. Indicated values are the mean of $\mathrm{T}$ divided by $\mathrm{N}$ and the standard error. $\mathrm{T}$ : tumor tissue; $\mathrm{N}$ :

Interestingly, although 5-hmC levels were found to be generally decreased in GCs compared to gastric mucosa, the reduction in the tumor portion was inversely correlated with the progression of the tumor portion in cases with extensive lymph node metastasis. Reduction of 5-hmC in the tumors was less in cases where the tumor had invaded further (T4) than in cases where the tumors were limited to the mucosa (so-called early cancer $\mathrm{T} 1$ ). In short, T4 and N3 cases (progressive cases) had larger amounts of 5-hmC (Fig. 4, $P<0.05$ ). The samples with stage I consistently had lower 5-hmC values than those with stage II and stage III (Figure S5, $P<0.05$ ). These findings together may suggest that the loss of 5-hmC in human GCs occurred in the early stage, at least in our cohort. More extensive studies using multiple cohorts will be needed to determine whether the loss of 5-hmC could become one of the markers representing some epigenetic nature of human gastric cancer in future.

Several enzymes influence DNA 5-hmC levels. To investigate the possible mechanism of 5-hmC reduction in GCs we quantified transcripts of 7 related genes using realtime RT-PCR. TETI-3, TDG and IDH2 were significantly reduced in the tumor portions in the GC cases. Downregulation of TET1 in particular was correlated with the tumor location of the GC. Surprisingly, down-regulation of $I D H 2$ was positively correlated with the tumor N classification and the tumor stage; thus, $I D H 2$ may play a role in the progress of GC. These findings are partially consistent with a recent study of melanoma. TET2 was capable of reestablishing the 5-hmC landscape in human melanoma cells. IDH2 increased the 5-hmC levels and prolonged tumor-free survival in a zebrafish melanoma model [11]. This evidence suggests that a deficiency of these enzymes may lead to one of the mechanisms of global 5-hmC reduction in GC. The next important finding was that the global 5-hmC levels were significantly and positively correlated with TET1 expression using a linear regression analysis, but not with TET2-3, TDG or IDH1-2. Additionally, our in vitro study confirmed that TETl has the normal tissue. One-way ANOVA with Tukey's post hoc test, $* P<$ 0.05. a 5-hmC levels and tumor T classification; b 5-hmC levels and tumor $\mathrm{N}$ classification; c 5-hmC levels and tumor stage

highest efficiency in global DNA 5-hmC modification in HEK293T cells among TET family genes. Together, the global loss of 5-hmC in GCs is mainly associated with the down-regulation of TET1 transcripts. Considering that all the TET family genes directly catalyze $5-\mathrm{mC}$ to $5-\mathrm{hmC}$ and that both TET2 and TET3 were down-regulated in our samples, we continue to speculate that TET2 and TET3 may also impact 5-hmC modifications in GCs, at least to some degree.

On the other hand, several known confounders are capable of changing 5-hmC levels in the human body; for example, vitamin $\mathrm{C}$ and hydroquinone are both capable of increasing 5-hmC levels in vitro [27, 28]. Lifestyle factors, such as food and medicine intake as well as cosmetics use, also cannot be excluded from factors that may influence the 5-hmC level in the human stomach. Estimations of the intake of particular diets based on a food frequency questionnaire and other descriptive methods used in epidemiology are often difficult, but a comprehensive analysis including lifestyle information will be a necessary challenge in this field.

According to previous literature [29-31], down-regulation of IDH2 in GC may inhibits other 2-oxoglutaratedependent dioxygenases, particularly hypoxia-inducible factor (HIF) prolyl hydroxylases [29, 30] and the JmjC domain containing histone demethylases [31]. Thus, IDH2 down-regulation in gastric cancer may provide various insights regarding the biology of and therapeutic strategies for GCs.

Another finding, the down-regulation of $T D G$ in gastric cancer, is likely to attract many investigators in the future. The functions of $T D G$, with the exception of its DNA glycosylase activity, are elusive and these functions are speculated to be relevant to $p 53$ [32] and Wnt Signaling [33].

Very recently, Yang et al. [12] reported that 5-hmC levels were markedly and negatively correlated with tumor invasion; that is, with the TNM stage and the number of lymph node metastases in GCs. They measured the 5-hmC level using a DNA dot blot assay and the down-regulation 
of TET1 transcripts was notably associated with a reduction of 5-hmC levels in GCs. The positive correlation between TET1 expression and 5-hmC levels found in our correlation analysis was consistent with their clinical findings and was reasonable considering the function of TET1. Some inconsistencies were present in that the correlation between the reduction of 5-hmC and progression indicators $(\mathrm{N}$ or $\mathrm{T}$ factors) of cancer was not clear in our subjects. This inconsistency might be related to the different analysis technologies, the limited number of cases, differences in the patient populations and other confounding factors. Our studies not only showed down-regulation of TET1 in our samples but also that of TET2, TET3, TDG and IDH2.

DNA mutation is another factor that influences protein activity. TET2 is mutationally inactivated in about $15 \%$ of human myeloid cancers [34]. However, a few mutations of TET1-3 have been found in solid cancers and no somatic mutation in TET1-3 has been found to be associated with the loss of 5-hmC in our samples. Given that our sequencing study was performed using a relatively small number of available samples we also validated our findings using the Catalogue of Somatic Mutations in Cancer (COSMIC) [35]. As of January 31, 2014, the mutation rate of TET1 was $0 \%(0 / 47)$, that of TET2 was $0 \%(0 / 47)$ and that of TET3 was $2.23 \%$ (1/47; p.L711L) in GCs.

Despite the small number of samples, our robust measurements of the DNA 5-hmC adduct and the in vitro analysis validated a decrease in 5-hmC in GCs, mainly due to TET1 down-regulation. In addition to the biological significance of $T D G$ and $I D H 2$ reduction in gastric cancer information on the local status of $5-\mathrm{hmC}$ in the genome will be the next important topic of research on GCs in terms of acquired DNA changes, including adductomics [36]. Further studies in a larger cohort are required to evaluate the relationship among 5-hmC, TET1, IDH2 and clinical characteristic such as the stage of GC.

Acknowledgments This work was supported by grants from the Ministry of Health, Labour and Welfare (19-19,10103838), the Japan Society for the Promotion of Science (23790396, 23790439, 25460476), the Ministry of Education, Culture, Sports, Science and Technology (MEXT) (S-001), an Extramural Collaborative Research Grant from the Cancer Research Institute, Kanazawa University, the National Cancer Center Research and Development Fund, the Center of Innovation Program of JST, MEXT, and the Smoking Research Foundation. We thank Prof. K. Seio (Tokyo Institute of Technology, Department of Life Science) for his kind information regarding the purification of 5-carboxy-2'-deoxycytidine.

\section{References}

1. Tahiliani M, Koh KP, Shen Y, Pastor WA, Bandukwala H, Brudno $\mathrm{Y}$, et al. Conversion of 5-methylcytosine to 5-hydroxymethylcytosine in mammalian DNA by MLL partner TET1. Science. 2009;324:930-5.

2. Kriaucionis S, Heintz N. The nuclear DNA base 5-hydroxymethylcytosine is present in Purkinje neurons and the brain. Science. 2009;324:929-30.

3. Ito S, D'Alessio AC, Taranova OV, Hong K, Sowers LC, Zhang Y. Role of Tet proteins in $5 \mathrm{mC}$ to $5 \mathrm{hmC}$ conversion, ES-cell selfrenewal and inner cell mass specification. Nature. 2010;466: 1129-33.

4. He YF, Li BZ, Li Z, Liu P, Wang Y, Tang Q, et al. Tet-mediated formation of 5-carboxylcytosine and its excision by TDG in mammalian DNA. Science. 2011;333:1303-7.

5. Reitman ZJ, Yan H. Isocitrate dehydrogenase 1 and 2 mutations in cancer: alterations at a crossroads of cellular metabolism. J Natl Cancer Inst. 2010;102:932-41.

6. Xu W, Yang H, Liu Y, Yang Y, Wang P, Kim SH, et al. Oncometabolite 2-hydroxyglutarate is a competitive inhibitor of alpha-ketoglutarate-dependent dioxygenases. Cancer Cell. 2011; 19:17-30.

7. Jin SG, Jiang Y, Qiu R, Rauch TA, Wang Y, Schackert G, et al. 5-Hydroxymethylcytosine is strongly depleted in human cancers but its levels do not correlate with IDH1 mutations. Cancer Res. 2011;71:7360-5.

8. Yang H, Liu Y, Bai F, Zhang JY, Ma SH, Liu J, et al. Tumor development is associated with decrease of TET gene expression and 5-methylcytosine hydroxylation. Oncogene. 2013;32:663-9.

9. Kraus TF, Globisch D, Wagner M, Eigenbrod S, Widmann D, Munzel M, et al. Low values of 5-hydroxymethylcytosine $(5 \mathrm{hmC})$, the "sixth base," are associated with anaplasia in human brain tumors. Int J Cancer. 2012;131:1577-90.

10. Hsu CH, Peng KL, Kang ML, Chen YR, Yang YC, Tsai CH, et al. TET1 suppresses cancer invasion by activating the tissue inhibitors of metalloproteinases. Cell Rep. 2012;2:568-79.

11. Lian CG, Xu Y, Ceol C, Wu F, Larson A, Dresser K, et al. Loss of 5-hydroxymethylcytosine is an epigenetic hallmark of melanoma. Cell. 2012;150:1135-46.

12. Yang Q, Wu K, Ji M, Jin W, He N, Shi B, et al. Decreased 5-hydroxymethylcytosine $(5-\mathrm{hmC})$ is an independent poor prognostic factor in gastric cancer patients. J Biomed Nanotechnol. 2013;9:1607-16.

13. Association Japanese Gastric Cancer. Japanese classification of gastric carcinoma: 3rd English edition. Gastric Cancer. 2011;14: $101-12$.

14. Lauren P. The Two Histological Main Types of Gastric Carcinoma: Diffuse and So-Called Intestinal-Type Carcinoma. An Attempt at a Histo-Clinical Classification. Acta Pathol Microbiol Scand. 1965;64:31-49.

15. Oshima H, Matsunaga A, Fujimura T, Tsukamoto T, Taketo MM, Oshima M. Carcinogenesis in mouse stomach by simultaneous activation of the Wnt signaling and prostaglandin E2 pathway. Gastroenterology. 2006;131:1086-95.

16. Globisch D, Munzel M, Muller M, Michalakis S, Wagner M, Koch S, et al. Tissue distribution of 5-hydroxymethylcytosine and search for active demethylation intermediates. PLoS One. 2010;5:e15367.

17. Sumino M, Ohkubo A, Taguchi H, Seio K, Sekine M. Synthesis and properties of oligodeoxynucleotides containing 5-carboxy-2'deoxycytidines. Bioorg Med Chem Lett. 2008;18:274-7.

18. Nomura Y, Haginoya N, Ueno Y, Matsuda A. Nucleosides and nucleotides. 161. Incorporation of 5-(N-aminoalkyl)carbamoyl2 -deoxycytidines into oligodeoxyribonucleotides by a convenient post-synthetic modification method. Bioconjug Chem. 1996;6:2811-6.

19. Itahara T. Oxidation of Cytosine and 5-Methylcytosine Nucleosides and 5-Methyl-2'-deoxycytidine 5'-Monophosphate with Peroxosulfate Ions. Chem Lett. 1991;1591-4. 
20. Ismael O, Shimada A, Hama A, Sakaguchi H, Doisaki S, Muramatsu $\mathrm{H}$, et al. Mutations profile of polycythemia vera and essential thrombocythemia among Japanese children. Pediatr Blood Cancer. 2012;59:530-5.

21. Seshagiri S, Stawiski EW, Durinck S, Modrusan Z, Storm EE, Conboy CB, et al. Recurrent R-spondin fusions in colon cancer. Nature. 2012;488:660-4.

22. Grasso CS, Wu YM, Robinson DR, Cao X, Dhanasekaran SM, Khan AP, et al. The mutational landscape of lethal castrationresistant prostate cancer. Nature. 2012;487:239-43.

23. Stephens PJ, Tarpey PS, Davies H, Van Loo P, Greenman C, Wedge DC, et al. The landscape of cancer genes and mutational processes in breast cancer. Nature. 2012;486:400-4.

24. Liu S, Wang J, Su Y, Guerrero C, Zeng Y, Mitra D, et al. Quantitative assessment of Tet-induced oxidation products of 5-methylcytosine in cellular and tissue DNA. Nucleic Acids Res. 2013;41:6421-9.

25. Cadet J, Wagner JR. TET enzymatic oxidation of 5-methylcytosine, 5-hydroxymethylcytosine and 5-formylcytosine. Mutat Res. 2013;

26. Ivanov M, Kals M, Kacevska M, Barragan I, Kasuga K, Rane A, et al. Ontogeny, distribution and potential roles of 5-hydroxymethylcytosine in human liver function. Genome Biol. 2013;14: R83.

27. Minor EA, Court BL, Young JI, Wang G. Ascorbate induces teneleven translocation (Tet) methylcytosine dioxygenase-mediated generation of 5-hydroxymethylcytosine. J Biol Chem. 2013;288: 13669-74.

28. Coulter JB, O'Driscoll CM, Bressler JP. Hydroquinone increases 5-hydroxymethylcytosine formation through ten eleven translocation 1 (TET1) 5-methylcytosine dioxygenase. J Biol Chem. 2013;288:28792-800.

29. Briere JJ, Favier J, Benit P, El Ghouzzi V, Lorenzato A, Rabier $\mathrm{D}$, et al. Mitochondrial succinate is instrumental for HIF1alpha nuclear translocation in SDHA-mutant fibroblasts under normoxic conditions. Hum Mol Genet. 2005;14:3263-9.

30. Selak MA, Armour SM, MacKenzie ED, Boulahbel H, Watson DG, Mansfield KD, et al. Succinate links TCA cycle dysfunction to oncogenesis by inhibiting HIF-alpha prolyl hydroxylase. Cancer Cell. 2005;7:77-85.

31. Shi Y. Histone lysine demethylases: emerging roles in development, physiology and disease. Nat Rev Genet. 2007;8:829-33.

32. Da Costa NM, Hautefeuille A, Cros MP, Melendez ME, Waters T, Swann P, et al. Transcriptional regulation of thymine DNA glycosylase (TDG) by the tumor suppressor protein p53. Cell Cycle. 2012;11:4570-8.

33. Xu X, Yu T, Shi J, Chen X, Zhang W, Lin T, et al. Thymine DNA glycosylase is a positive regulator of Wnt signaling in colorectal cancer. J Biol Chem. 2014

34. Delhommeau F, Dupont S, Della Valle V, James C, Trannoy S, Masse A, et al. Mutation in TET2 in myeloid cancers. N Engl J Med. 2009;360:2289-301.

35. Forbes SA, Bindal N, Bamford S, Cole C, Kok CY, Beare D, et al. COSMIC: mining complete cancer genomes in the catalogue of somatic mutations in cancer. Nucleic Acids Res. 2011;39:D945-50.

36. Balbo S, Turesky RJ, Villalta PW. DNA adductomics. Chem Res Toxicol. 2014 